\title{
Simulation Agent-Based Model to Demonstrate the Transmission of COVID-19 and Effectiveness of Different Public Health Strategies
}

\author{
Yixing Wang ${ }^{1 \dagger}$, Hainan Xiong ${ }^{2 \dagger}$, Sijie Liu $^{3 \dagger}$, Ara Jung ${ }^{4,5}$, Trish Stone ${ }^{4}$ and \\ Leanne Chukoskie ${ }^{4,5 *}$
}

${ }^{1}$ Department of Computer Science and Engineering, University of California, San Diego, San Diego, CA, United States, ${ }^{2}$ Department of Mathematics, University of California, San Diego, San Diego, CA, United States, ${ }^{3}$ Hallııoğlu Data Science Institute, University of California, San Diego, San Diego, CA, United States, ${ }^{4}$ Qualcomm Institute, University of California, San Diego, San Diego, CA, United States, ${ }^{5}$ Department of Physical Therapy, Movement and Rehabilitation Science, Bouvé College of Health, Games and Design, College of Arts, Media, and Design, Northeastern University, Boston, MA, United States

OPEN ACCESS

Edited by:

Mina C. Johnson-Glenberg, Arizona State University, United States

Reviewed by:

Saturnino Luz,

University of Edinburgh,

United Kingdom

Despina Michael-Grigoriou, Cyprus University of Technology,

Cyprus

\section{${ }^{*}$ Correspondence:}

Leanne Chukoskie

I.chukoskie@northeastern.edu

${ }^{\dagger}$ These authors have contributed equally to this work and share first authorship

Specialty section:

This article was submitted to

Human-Media Interaction,

a section of the journal

Frontiers in Computer Science

Received: 15 December 2020

Accepted: 18 August 2021

Published: 20 September 2021

Citation:

Wang Y, Xiong H, Liu S, Jung A, Stone $T$ and Chukoskie L (2021) Simulation Agent-Based Model to Demonstrate the Transmission of

COVID-19 and Effectiveness of

Different Public Health Strategies.

Front. Comput. Sci. 3:642321.

doi: 10.3389/fcomp.2021.642321
COVID-19 has changed the world fundamentally since its outbreak in January 2020. Public health experts and administrations around the world suggested and implemented various intervention strategies to slow down the transmission of the virus. To illustrate to the general public how the virus is transmitted and how different intervention strategies can check the transmission, we built an agent-based model (ABM) to simulate the transmission of the virus in the real world and demonstrate how to prevent its spread with public health strategies.

Keywords: COVID-19, agent-based model, public health strategies, epidemic interventions, simulation, unity

\section{INTRODUCTION}

As a novel coronavirus, named SARS-CoV-2, reached epidemic proportions around the world, policymakers, public health experts and different levels of administrative organizations recommended various kinds of intervention strategies to slow down the outbreak of the virus. This urgent work occurred at a rapid pace and was widely reported, yet the general public did not uniformly enjoy an understanding of the basics of viral transmission in general or the specifics of COVID-19 in particular that would help them make informed decisions about their own actions or to support their community in lowering viral transmission. Since an effective public health response relies in part on personal behavior change (Southwell, et al., 2020), and in the case of COVID-19, such changes needed to occur amidst substantial misinformation about the virus (Brennan, et al., 2020), we were intrigued by the problem of providing accessible tools for the public to learn about viral transmission. We sought to create a tool that would provide users in the public at large with the opportunity to explore for themselves how population uptake of different public health strategies affects viral transmission. We built an agent-based model using Unity that uses data structures to represent individuals in a community with rules governing the interaction between individuals to simulate the process of viral transmission. The simulation's interactive interface allows users to change parameters that effect transmission, including intervention strategies. By changing parameters at different points in the simulation, users can visualize how different public health strategies affect the transmission of COVID-19 (Hoertel, et al., 2020). Leveraging Unity's built-in rendering system, users can visually monitor a simulated community epidemic situation as it unfolds, make decisions about what strategies to implement and observe simulated outcomes. To gain some 
understanding of how users engaged the simulation, we conducted a small qualitative user study with a sample of high school and college students. The small user group supported the contention that the simulation offers an accessible opportunity to explore different viral spread mitigation strategies as well as the effects on the timing in using these strategies in terms of curbing community transmission.

\section{MATERIALS AND METHODS}

\section{Agent-Based Modeling}

We found that most of the models aiming to quantify the effectiveness of different public health intervention strategies for COVID-19 fell into one of the two general categories: equation-based models or agent-based models. Compared with agent-based models, equation-based models apply a "top-down" structure. Research using equation-based models typically choose compartmental models that are commonly used in epidemiology such as SIR or SEIR models to simulate infectious diseases. Within these models different parameters such as transmission rate or contact rate can be manipulated to simulate the effectiveness of different control strategies (Matrajt and Leung, 2020; Rocklöv, et al., 2020). Agent-based models, on the other hand, apply a "bottom-up" structure. In the usage applied to epidemics, they start from using data structures to represent each individual in the community and then let the individuals interact with each other (thus spreading the virus) based on a selected set of rules (Eubank et al., 2004; Cliff et al., 2018; Chinazzi et al., 2020; Hoertel et al., 2020). The application of different intervention strategies can change those rules accordingly. By engaging these simulations, users can manipulate the parameters that confer probability of infection as well as aspects of different intervention strategies applied and observe the results.

The equation-based models we examined dealt with one or two intervention strategies by adjusting coefficients. We found it challenging to fit various intervention strategies reasonably into one equation-based model to quantify the effectiveness of many strategies applied concurrently (Lekone and Finkenstädt, 2006; Biswas et al., 2014; Cuevas, 2020; Rocklöv et al., 2020). Moreover, adding parameters reflecting the implementation of epidemic intervention strategies increases the complexity of an equationbased model without necessarily making the model more valid and accurate when making decisions (Roda et al., 2020). In a "bottom-up" model, the effect of a strategy on the transmission of the virus is reflected on an individual level (Eubank et al., 2004; Cliff et al., 2018; Perkins et al., 2019; Chinazzi et al., 2020). Implementing one or several strategies will change the person-toperson transmissibility differently and the individual change will accumulate to have an overall impact on the entire simulation. Additional reasons for implementing an agent-based model are that it can involve heterogeneity which categorizes individuals with different roles representing their possible different responses and actions in the system and take into account the dynamics of agents across space and time (Cliff, et al., 2018; Perkins, et al., 2019; Cuevas, 2020). Through the adjustment of simulation parameters, the agent-based model enabled us to visually show users the simulated effects of differential uptake of public health measures on virus transmission within the city arrangement.

\section{Quantifying Transmissibility in Simulation}

Our simulation is based on the following stochastic epidemic transmission model (Hoertel et al., 2020). In epidemiology, basic reproduction number, denoted as $\mathrm{R}_{0}$, is interpreted as the expected number of cases which are directly generated by one case in a certain population. The formula of basic reproduction number is:

$$
R_{0}=\beta \tau
$$

$\beta$ is the number of infection-producing contacts per unit time, and $\tau$ is the mean infectious period. In our model, for simplicity, $\tau$ can be regarded as the expected number

$$
E(X)=n p
$$

where $\mathrm{X}$, a random variable, is the number of persons an infected person can transmit virus to per unit time, assuming $\mathrm{X}$ follows a binomial distribution, and $\mathrm{n}$, the number of other individuals a person can meet per unit day. Hence, the person-to-person transmissibility, $p$, is derived from basic reproduction numbers $\mathrm{R}_{0}$, assuming

$$
R_{0}=E(X) \tau=n p \tau
$$

To compare the simulated effectiveness of different intervention strategies we quantify each strategy's effect on the extent of transmissibility between individuals, $p$. For example, we assume that masks can reduce the percent of disease-inducing particles a person inhales or exhales $(\pi)$. This makes the personto-person transmissibility equal to $(1-\pi)^{\star} p$. In a different example, a stay-at-home order strategy can lower the number of individuals met per day $(n)$. Assuming that a stay-at-home order can lower a percent of $n$, then the new $n$ will be $(1-\alpha)^{*} n$. Hence the expected number of cases, $\mathrm{E}(\mathrm{Y})$, when both masks and a stay-at-home orders are in effect, can be represented as

$$
E(Y)=(1-\alpha) n(1-\pi) p \tau
$$

If no strategies are implemented, then $\alpha$ and $\pi$ are both 0 . All variables are adjustable in the simulation.

\section{Simulation Design}

The simulation was implemented using the popular game development engine Unity version 2019.4.18f1c1 (Unity Technologies (2020), San Francisco). We represent each individual as a collidable sphere object and paint these spheres with different colors to represent different health status conditions, with green standing for "healthy," orange for "infected," red for "detected," and blue for "recovered." The simulation follows a timeline starting from day 0 at 12:00 a.m. Currently there are three types of building structures: individuals' residences (houses), workplaces, and hospitals. Based on these three building structures, the simulation follows a simple societal model: individuals leave their houses at some point from 6:00 a.m. to 7:00 a.m, heading to their workplaces that are randomly assigned, and go back to their houses from the workplaces at some point 
during 6:00 p.m. and 7:00 p.m. For any of their trips between building structures, each individual will follow a random route consisting of several roads on the map. Individuals can only move in one of the four directions in the map defined as "left," "right," "up" and "down." So accordingly, roads will only have directions of "left-right" and "up-down." We make individuals randomly move in one of the four directions when they are inside their houses or workplaces, in order to enable them to have contact with other people currently in the same building. To simply simulate individual social interactions, the sphere objects that represent individuals can collide with each other. Collisions won't change sphere objects' original moving directions. In real life, an individual may or may not interact with many different people in a day, but the interaction numbers tend to be low (Zhaoyang et al., 2018). In our simulation instances of interaction can get quite high, so we have a parameter that sets a cap on at most how many collisions are counted as valid for each individual per hour.

When a healthy individual interacts or collides with an infected individual, there is a possibility that the healthy individual can get infected (health status indicator changed from green to orange). This possibility is called the "Transmission possibility per contact" in the simulation and is a parameter the user can set. From the point that an individual becomes infected, we set up a timer for that individual to keep track of how long they have carried the virus. We compared that length with a preset "incubation time." If an individual has carried the virus longer than the incubation time, we assume a symptom onset for them so that the agent will decide whether to go to a hospital for a test. The probability of going for a test is also a parameter that users can manipulate. Once an infected individual decides to go for a test, regardless of their current location, they will move to a random hospital on the map. Once an infected individual arrives at any hospital, their status will change from "infected" to "detected" (health status indicator changed from orange to red) and they will remain in the hospital. After passing a period of time which we set as "average cure time," the detected individual will either die or recover. The possibility of a person dying can also be set as a parameter called "fatality rate." Recovered people will leave the hospital and go to their houses automatically and continue their "house-work place" loop again. For simplicity reasons, recovered people will neither get infected again nor infect other healthy people.

During any stage of the simulation, the player can change the public health strategies that they want to apply. Currently we only have two strategies implemented--the "Put on masks" order and the "Stay -at -home" order. If the player selects the "Put on masks" order, there is a possibility for each individual to obey this order to "put on a mask." We simulate this effect by lowering the transmission possibility between infected and healthy people with their face masks on. If the player selects the "Stay -at -home" order, there is a possibility for each individual to obey this order to stay at their house all day, without commuting between their workplace and house anymore.

The simulation is highly customizable and the user can simulate the spread process of many viruses and infectious diseases other than just COVID-19. The simulation allows the user to change the following parameters (See Supplementary Material):
- In terms of the attributes of virus itself

- Transmission possibility per contact--the possibility for a healthy person getting infected each time they contact an infected person;

- Initial Infected Percentage--the percentage of population that get infected at the beginning of the simulation;

- Fatality rate--the ratio of deaths over the total number of infected cases;

- Average incubation time--the average time in hours between a person get infected and the onset of the symptom. After the symptom onset, the infected person will realize they are infected;

- Average cure time--the average time in hours it takes an infected person to recover in a hospital;

- In terms of people's general behavior in the simulation

- Average number of contacts with other people per hour--the maximum number of valid contacts for each person per hour. In other words, only the first this number of contacts per hour a person has with others can generate infections.

- People's tendency to have a test after they have symptoms-possibility for an infected person to go to the hospital for a test after they spot a symptom onset;

- In terms of public health strategy parameters

- Transmission possibility per contact with people wearing masks--the reduced transmission possibility per contact after putting "Put on masks" strategy into effect.

- People's tendency to wear a mask--the possibility of each person obeying the "Put on masks" order;

- People's tendency to obey the "Stay--home" order.

- And overall, the user can change the size of the population.

The simulation itself is free to download and run. A video description of the project, as well as the code for the simulation is also available on the project website (see Supplementary Material). The visualization of simulation data is conducted via ggplot2 (Wickham, 2016) from $\mathrm{R}$ 3.6.3 (R Core Team, 2020). The simulation data is collected by outputting data into local csv files. The write-out function is implemented within the source code (also available, see Supplementary Material).

\section{User Experience Focus Group}

The simulation was shared with a convenience sample of seven students (ages 16-21), all living in the United States in April 2021. The study was run via Zoom, with students using their PC and preferred web browser while sharing screen. Each student participant was given $10 \mathrm{~min}$ to interact with the simulation and the https://pongcenter.itch.io/anti-plague page while the researcher muted and disabled her camera, unmuting only to ask or answer questions. This was set in hopes of simulating a natural, at-home environment, close to how the users would be using this simulation. The users were then asked a list of questions to answer related to any observations about the simulation and their understanding of it (see survey report in Supplementary Material). The interview was covered by a protocol approved by UC San Diego's Human Subjects Protection Program. All participants provided consent and/or assent to participate (in the case on minors) in this remote interview. 


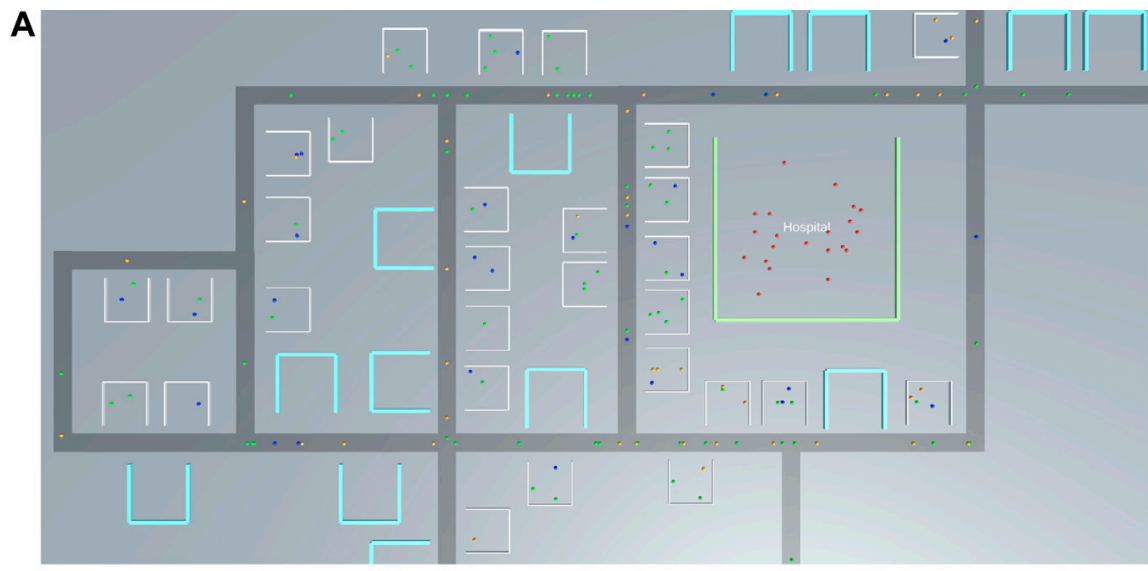

The text for the epidemic status in the top-left corner is cropped here due to limited space: Day 0, 18: 46; Population: 215; Infected: 120; Detected: 59; Recovered: 36; Deaths: 2

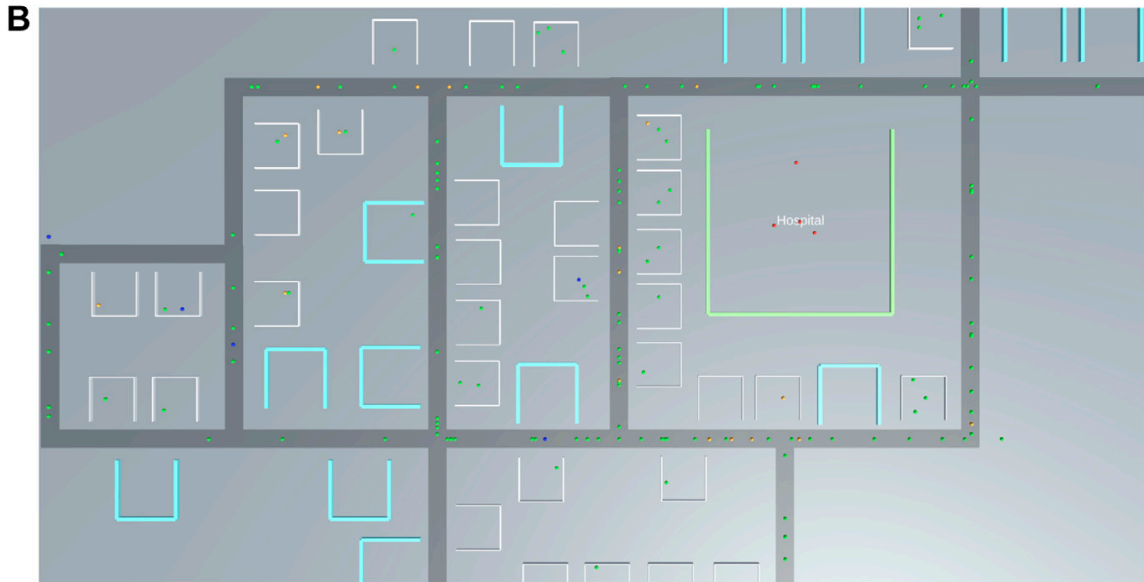

\section{Day 0, 18: 24; Population: 215; Infected: 38; Detected: 16; Recovered: 11; Deaths: 1}

C Transmission without Intervention Under Different R0s and Population Densities

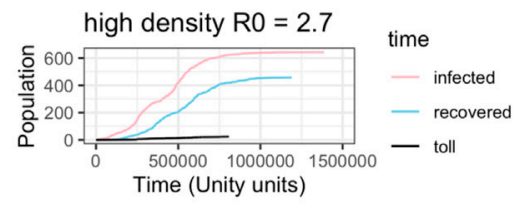

high density $\mathrm{R} 0=1.6$
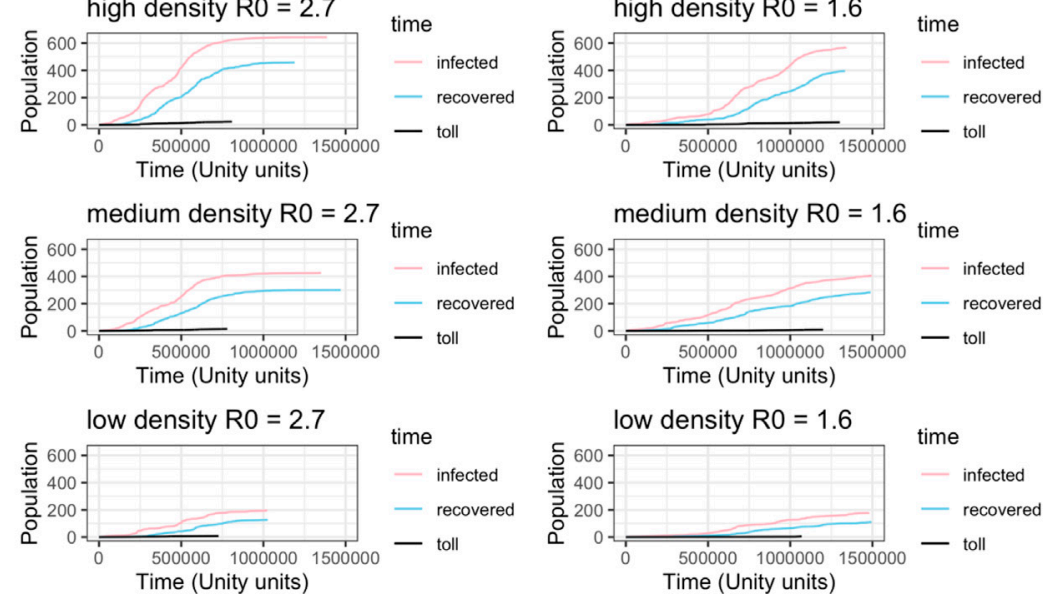

FIGURE 1 | The results of different simulations given different parameter settings: (A) screen shot of the simulation given $R 0=2.7$ (B) same as in subplot a, but with $\mathrm{RO}=1.6$ (C) shows how population density changes the rate of infection over time for the two different R0 values shown in subplots a and b. Lines in each subplot show time course of infected, recovered and death toll count for each set of conditions. 


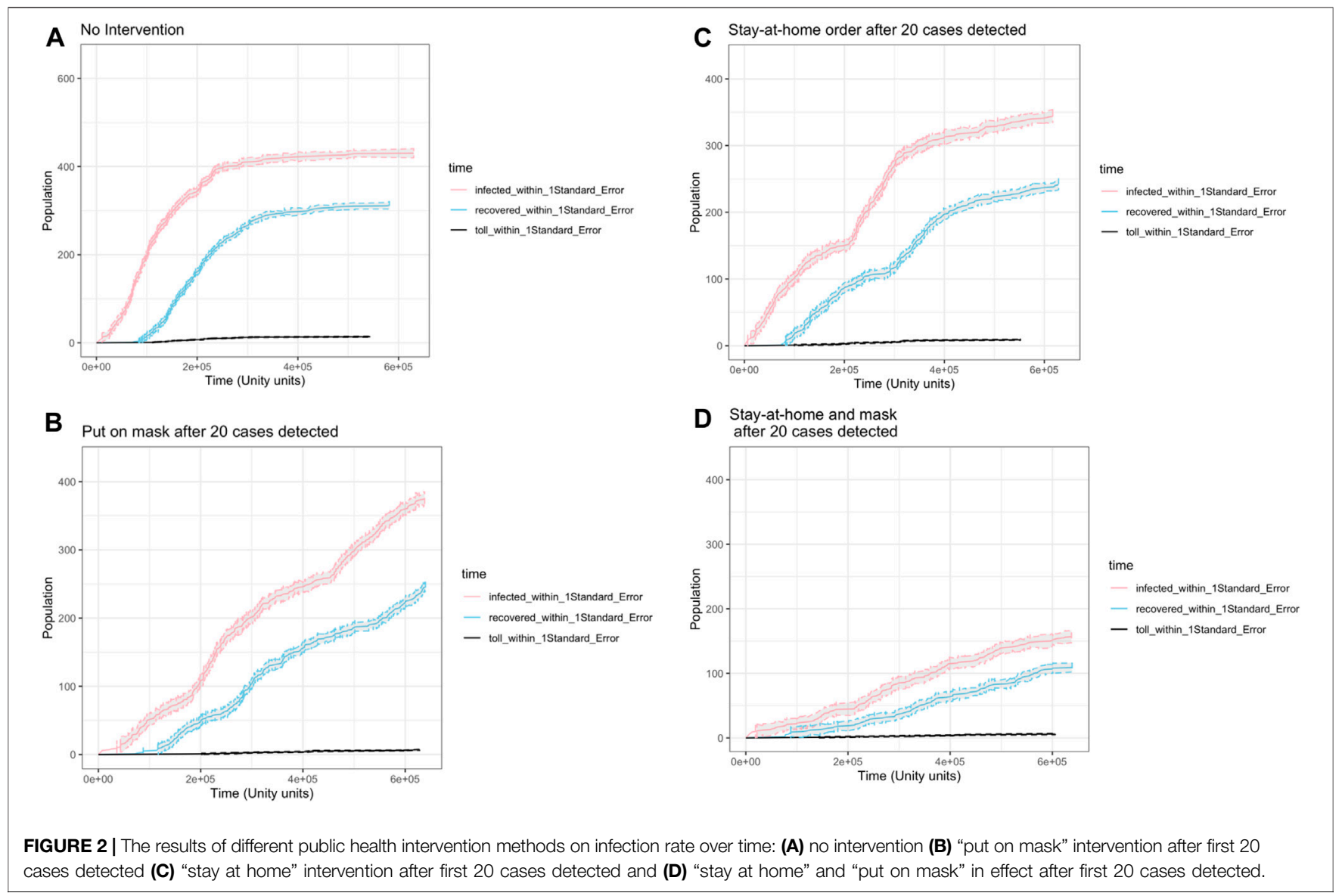

\section{RESULTS}

To examine the outcomes of our simulation, as well as the effects of the public health strategies we implement, we ran simulations in different scenarios to observe the outcomes. A statistical comparison between our simulation and real world data are not feasible at this point for multiple reasons, but particularly due to the small size of the simulated population in comparison with real world populations. The data shown here offer an example of what a user might observe under different parameter scenarios when using the simulation. To speed up the simulation, we used Unity time units instead of the realworld time units. In the simulations shown below, one Unity time unit represents $1 \mathrm{~s}$, and 50 Unity time units will pass for each frame.

\section{Transmission Under Different Population Density and Different $\mathbf{R}_{\mathbf{0}}$}

We simulated two different $\mathrm{R}_{0}$ in low density community. In Figure $1 \mathrm{~A}$ the $\mathrm{R}_{0}$ is set to 2.7 and in Figure $\mathbf{1 B}$ the $\mathrm{R}_{0}$ is set to 1.6 while the population size is set to 215 in both cases. The two different $\mathrm{R}_{0}$ scenarios generate substantially different results: in Figure 1A over half of the community have been infected while in Figure 1B approximately over one seventh of the population have been infected. This simulation comparison illustrates the idea that given the same community population density, a disease with higher basic reproduction number is more contagious and results in a larger number of infections. To better illustrate the change rate of infected population, recovered population, and death toll, we plot them as curves in Figure 1C. Figure 1C shows simulation results under different population density and different reproduction number $\mathrm{R}_{0}$ (Al-Raeei, 2021). Given the number of residential buildings is stationary per simulation, the population density is modified by changing the population number each household can generate. High density is set to have 15 agents generated per household. Medium density is set to have 10 agents generated per household. Low density is set to have five agents generated per household. The average number of agents each agent can encounter per hour is set to $1 . \tau$ is set to a single Unity time for the sake of simplicity. The probability of an infected agent transmitting virus to other agents per contact is set to be 0.0667 under $R_{0}=1.6$. The probability of an infected agent transmitting virus to other agents per contact is set to 0.1125 under $R_{0}=2.7$. The curves in the plots stop when there is no change in the rate anymore. The simulation results show that the transmission is fastest when both the population density and basic reproduction number are high. In the latter simulations, the total population of agents in the 


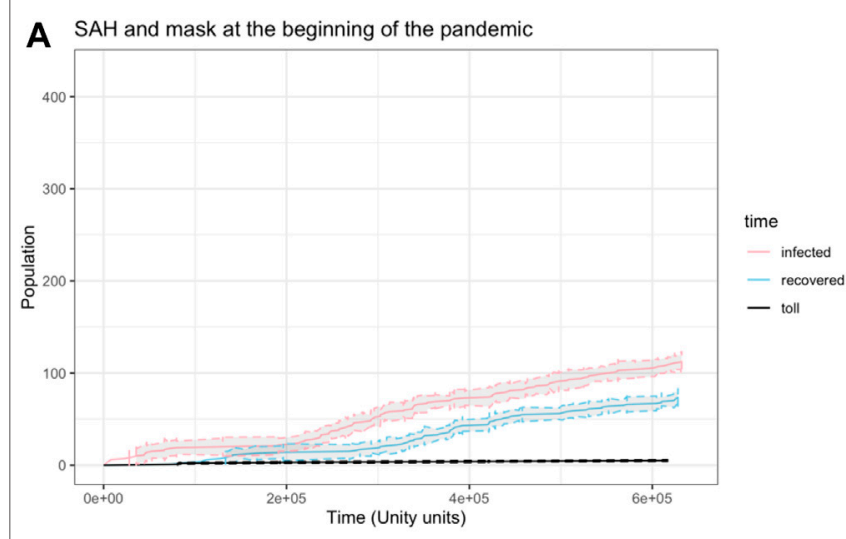

B SAH and mask in the middle of the pandemic

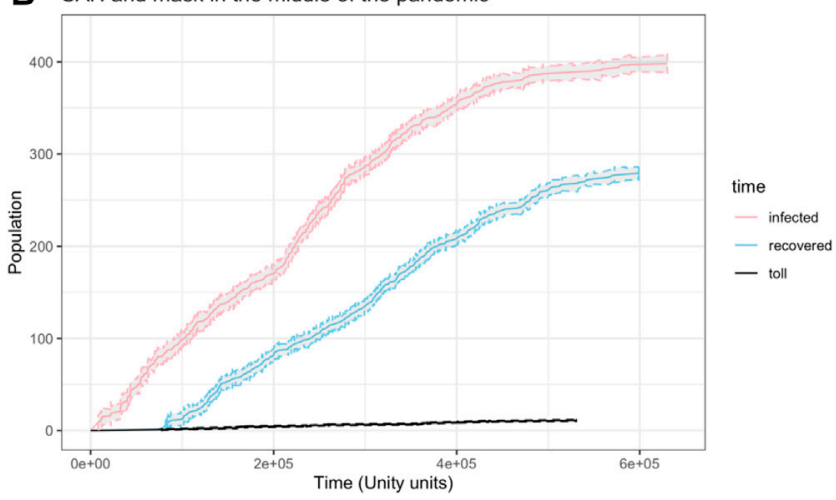

FIGURE 3 | The results of implementing both public health intervention methods on infection rate at different phases of the pandemic: (A) both interventions implemented at the beginning phase (B) both interventions applied in a later phase of the pandemic.

simulation is set up to 430 , using a medium density setting. Each time we restarted the simulation the number of initial infected persons was set to 8 .

\section{Transmission Under Different Intervention Approaches}

Figure 2 compares a no intervention scenario to three different public health intervention scenarios: 1) mask order after 20 infected persons detected in the simulation, 2) stay-at-home order after 20 infected persons detected, and 3) initiating both mask and stay-at-home measures after 20 infected persons have been detected. Figure 2A shows that by $2 \mathrm{e}+05$ Unity time units elapsed there are already over 340 persons infected, more than $79 \%$ of the population. The infection number breaks over 400 at approximately $2.7 \mathrm{e}+05$ Unity time units elapsed. Figure $3 \mathbf{B}$ shows that adding a mask order, the curve is slowed down and by the end of $6 e+05$ Unity time, the infected population is less than 400. Figure $2 \mathrm{C}$ shows that under the stay-at-home mandate by the end of $6 e+05$ Unity time, the infected population is less than 350 , showing that stay-at-home order is more effective than put-onmask alone. Figure 2D shows implementing two strategies together is more effective than either single strategy alone.
We also simulated the impact of implementing public health strategies at different phases of the pandemic, using a medium density setting. To simulate the effects of implementing public health strategies during the beginning phase of the pandemic, we initiated strategies right after the simulation started. To simulate the effects of delayed implementation of public health strategies, we initiated strategies only after 50 individual agents have been infected. Figure 3 shows the simulated reduction in infections by implementing public health strategies at the beginning (Figure 3A) versus later in the pandemic (Figure 3B).

\section{User Experience Focus Group}

Our user experience qualitative study provided insights into how a small sample of high school and college students engaged with the simulation through both direct observation and questions about the experience (see Supplementary Material). It was clear that users understood that the simulation's primary purpose was to reflect a pandemic setting and observe effects of public health interventions on the rate of infection. These users reported finding the simulation easy to use, although it was observed that some users skipped the instructions and found themselves going back to the instructions for more information once they got further along. Some users made suggestions for further features or functionality and three wanted to spend more than the allotted 10 min with the simulation in this study (all were provided with the link to explore further on their own). The visualization aspect was appreciated as several students noted that it was "cool" to see the immediate effect of a simulation parameter change on viral transmission.

\section{DISCUSSION}

From the time that SARS-CoV-2 was initially detected and developed into a global pandemic, different public health strategies were implemented around the world, with different levels of population uptake and efficacy. Within the United States alone, there have been many different approaches to mitigate the spread of the virus, and quite varied adherence to these recommended approaches, with concomitant results in changing rates of infection. The type of public health mitigation strategy, timing of implementation, and the uptake of the strategy in the population all impacted the rate of transmission in the real world (Bremmer, 2020; Gibney, 2020). The presumed large number of asymptomatic cases demanded ubiquitous and regular testing as well as contact tracing to detect infected cases and has been simulated using an SEIR model that sought to "visualize the invisible" (Peirlinck et al., 2020). Multiple efforts have been invested in visualizing the spatial distribution of viral transmission and its frequency through infographics (see Leung et al. (2020) for review), however, there are key concepts underlying viral transmission that are important to understand for making science-based decisions that impact public health and these remain challenging to grasp for the public at large. We sought to create an accessible tool for users in the public sphere to explore different aspects of viral transmission in a simulated 
environment along with the effects of applying simulations of common public health interventions. Although our efforts were small in scale and the evaluation of these were qualitative in nature, our user group found the tool to be easy to use, and we observed that the trends in our simulation in many ways mirrored trends observed in viral transmission during COVID-19. It is important to note, however, that this simulation has not been validated with real-world data and therefore should not be used in making health-related decisions.

Visualizations from the simulation shown in Figure 1 illustrate an expected result: that without any interventions, the transmission of a virus is quicker when its basic reproduction number $R_{0}$, which measures the level of contagiousness, is high. The rate of transmission is determined by the population density as well. In our simulation, as the population density increased, the infection rate increases rapidly and reaches its highest number quickly. By making multiple aspects of viral transmission adjustable through parameters in our simulation, we provide users with a way to engage with these concepts and observe the effects.

Data from the simulation shown in Figure 2A illustrates that the virus is transmitted rapidly at the beginning of the pandemic and once approximately 90 percent of the population are either infected, or immune, the rate of transmission slows down. Under the same environment and setting we observe that the intervention efforts applied alone (Figures 2B,D) and especially together (Figure 2C) slow down simulated transmission rate dramatically.

The pandemic has demanded a shift in educational strategies in all levels, from prior in-person instruction to predominantly remote learning. This situation has emphasized the need for hands-on learning opportunities that can be conducted at home. In addition, in spite of the global spread of the COVID19 pandemic, we have not necessarily enjoyed a similar spread in understanding the nature of viral transmission (Brennen et al., 2020). Simulation also has a substantial role to play not only in learning practical skills but also in arenas such as laboratory learning and practicing simulated medical and dental procedures (Tabatabai, 2020). There is also considerable value in using simulations to reconsider work proximity, pedestrian traffic flow and building density with respect to education (Sutton and Jorge, 2020). Simulations that can be conducted at home, on a personal computer, have potential for helping people understand the nature of viral transmission and their individual role in minimizing it. We believe our simulation also has value in terms of inviting users to consider the science behind this sort of simulation-based planning as well as learning more about the simulation environment itself as it can be adapted and used for other purposes. Our small qualitative user experience study observations suggest that users do find the simulation to be engaging, visually interesting and informative. A much larger quantitative study would be needed with assessments conducted both pre- and post-simulation engagement to understand what students take away from the simulation in terms of knowledge gained, behaviors changed, or assumptions challenged.

We would also like to note that although this simulation was designed for informational purposes, it is possible that one might compare the simulation results with real world data if the population size of the simulation were dramatically increased. A rigorous statistical comparison with epidemiological data is outside the goals of our simulation, and the computational power available to us. We designed the simulation such that each agent was fully-instantiated and existed at home, work, hospital or in transit between those sites. Collisions could probabilistically cause infection in any of those places, but this design choice created a computational cost. It is not feasible to simulate a large population size with a standard computer. We did not design our simulations to model real-world data statistically, although with a supercomputer this may be feasible. However, our simulation can be a practical aid for learning about viral transmission and how different public health measures might effect the transmission of the virus. Although this simulation is developed with a goal to simulate COVID-19, the parameters such as person-to-person transmissibility, incubation time, fatality rate, etc., can be manipulated to simulate other contagious diseases as well.

\section{FUTURE DEVELOPMENTS}

The simulation we developed serves only as a template or a prototype for future development. By leveraging the power of Unity's user interface (UI) engine, it is relatively easy and convenient for developers, including novice developers, to extend the simulation's features and functionality, thus increasing the complexity of simulations to make them more realistic. In the future iterations, we plan to build a UI that allows players to edit the map by adding or deleting different building structures and moving their positions. For example, we can develop additional types of building structures such as public entertainment venues and public commuting centers to simulate their effects on viral transmission. We can also simulate the effects of viral transmission on a particular population's unique age distribution by applying differential susceptibilities to individual of different ages. Finally, we plan to implement more intervention strategies, specifically large-scale vaccination and contact tracing. Although these two strategies are challenging to implement in the real world, they are relatively easy to integrate into the current model by their nature since the model monitors every individual agent and every contact taking place in the simulations. We believe that with the future improvement on algorithms and data management, more computational resources, more customizable maps and more intervention strategies added, our simulation program can provide users with a learning tool that helps them better understand the nature of viral transmission under many different conditions. Future developments should also include a larger study to understand how engagement of the simulation impacts user understanding of viral transmission and its impact on public health.

\section{DATA AVAILABILITY STATEMENT}

The datasets presented in this study can be found in online repositories. The names of the repository/repositories and accession number(s) can be found in the article/Supplementary Material. 


\section{AUTHOR CONTRIBUTIONS}

Project Design: YW, HX, SL, AJ. Analysis: HX, YW, AJ. Software Development: YW, HX. Supervision: TS, LC. Writing: YW, HX, SL, LC.

\section{FUNDING}

This research was part of the Experiential Learning Academy academic internship program at the Qualcomm Institute, UC San Diego. A portion of the support for this program was provided by the Legler Benbough Foundation. This work was an extension of an initial academic internship project at

\section{REFERENCES}

Al-Raeei, M. (2021). The basic reproduction number of the new coronavirus pandemic with mortality for India, the Syrian Arab Republic, the United States, Yemen, China, France, Nigeria and Russia with different rate of cases. Clin. Epidemiol. Glob. Health 9, 147-149. doi:10.1016/j.cegh.2020.08.005

Bremmer, I. (2020). The Best Global Responses to COVID-19 Pandemic. Time. https:// time.com/5851633/best-global-responses-covid-19/ UpdatedFebruary 23, 2021).

Brennen, J. S., Howard, P. N., Nielsen, R. K., and Nielsen, R. K. (2020).Balancing Product Reviews, Traffic Targets, and Industry Criticism: UK Technology Journalism in Practice. Journalism Pract., 7. 1-18. doi:10.1080/ 17512786.2020.1783567

Chinazzi, M., Davis, J. T., Ajelli, M., Gioannini, C., Litvinova, M., Merler, S., et al. (2020). The effect of travel restrictions on the spread of the 2019 novel coronavirus (COVID-19) outbreak. Science 368 (6489), 395-400. doi:10.1126/science.aba9757

Cliff, O. M., Harding, N., Piraveenan, M., Erten, E. Y., Gambhir, M., and Prokopenko, M. (2018). Investigating spatiotemporal dynamics and synchrony of influenza epidemics in Australia: An agent-based modelling approach. Simulation Model. Pract. Theor. 87, 412-431. ISSN 1569-190X. doi:10.1016/j.simpat.2018.07.005

Cuevas, E. (2020). An agent-based model to evaluate the COVID-19 transmission risks in facilities. Comput. Biol. Med. 121, 103827. doi:10.1016/j.compbiomed.2020.103827

Eubank, S., Guclu, H., Anil Kumar, V. S., Marathe, M. V., Srinivasan, A., Toroczkai, Z., et al. (2004). Modelling disease outbreaks in realistic urban social networks. Nature 429 (6988), 180-184. doi:10.1038/nature02541

Gibney, E. (2020). Whose coronavirus strategy worked best? Scientists hunt most effective policies. Nature 581, 15-16. doi:10.1038/d41586-020-01248-1

H. A. Biswas, M., Paiva, M. T. L., T. Paiva, L., and de Pinho, M. (2014). A SEIR model for control of infectious diseases with constraints. Math. Biosciences Eng. 11 (4), 761-784. doi:10.3934/mbe.2014.11.761

Hoertel, N., Blachier, M., Blanco, C., Olfson, M., Massetti, M., Rico, M. S., et al. (2020). A stochastic agent-based model of the SARS-CoV-2 epidemic in France. Nat. Med. 26, 1417-1421. doi:10.1038/s41591-020-1001-6

Lekone, P. E., and Finkenstädt, B. F. (2006). Statistical Inference in a Stochastic Epidemic SEIR Model with Control Intervention: Ebola as a Case Study. Biometrics 62 (4), 1170-1177. doi:10.1111/j.1541-0420.2006.00609.x

Leung, C. K., Chen, Y., Hoi, C. S., Shang, S., Wen, Y., and Cuzzocrea, A. (2020). Big Data Visualization and Visual Analytics of COVID-19 Data. Proceedings of the 2020 24th International Conference Information Visualisation (IV) Melbourne, Australia, September 7-11, 2020 IEEE, 415-420.

Matrajt, L., and Leung, T. (2020). Evaluating the Effectiveness of Social Distancing Interventions to Delay or Flatten the Epidemic Curve of Coronavirus Disease. Emerg. Infect. Dis. 26 (8), 1740-1748. doi:10.3201/eid2608.201093

Peirlinck, M., Linka, K., Sahli Costabal, F., Bhattacharya, J., Bendavid, E., Ioannidis, J. P. A., et al. (2020). Visualizing the invisible: The effect of asymptomatic
Qualcomm Institute conducted by undergraduate students from University of California, San Diego. This work was supported by the Power of Neurogaming Center (PoNG) and Qualcomm Institute at the University of California, San Diego. A portion of the support for this program was provided by the Legler Benbough Foundation.

\section{SUPPLEMENTARY MATERIAL}

The Supplementary Material for this article can be found online at: https://www.frontiersin.org/articles/10.3389/fcomp.2021.642321/ full\#supplementary-material transmission on the outbreak dynamics of COVID-19. Comp. Methods Appl. Mech. Eng. 372, 113410. doi:10.1016/j.cma.2020.113410

Perkins, T. A., Reiner, R. C., España, G., ten Bosch, Q. A., Verma, A., Liebman, K. A., et al. (2019). An agent-based model of dengue virus transmission shows how uncertainty about breakthrough infections influences vaccination impact projections. Plos Comput. Biol. 15 (3), e1006710. doi:10.1371/journal.pcbi.1006710

R Core Team (2020). R: A language and environment for statistical computing. Vienna, Austria: R Foundation for Statistical Computing. URL https://www.R-project.org/.

Rocklöv, J., Sjödin, H., and Wilder-Smith, A. (2020). COVID-19 outbreak on the Diamond Princess cruise ship: estimating the epidemic potential and effectiveness of public health countermeasures. J. Trav. Med. 27 (3). doi:10.1093/jtm/taaa030

Roda, W. C., Varughese, M. B., Han, D., and Li, M. Y. (2020). Why is it difficult to accurately predict the COVID-19 epidemic. Infect. Dis. Model. 5, 271-281. doi:10.1016/j.idm.2020.03.001

Southwell, B. G., Kelly, B. J., Bann, C. M., Squiers, L. B., Ray, S. E., and McCormack, L. A. (2020). Mental models of infectious diseases and public understanding of COVID-19 prevention. Health Commun. 35 (14), 1707-1710. doi:10.1080/10410236.2020.1837462

Sutton, M. J., and Jorge, C. F. B. (2020). Potential for radical change in Higher Education learning spaces after the pandemic. Jalt 3 (1). doi:10.37074/jalt.2020.3.1.20

Tabatabai, S. (2020). Simulations and virtual learning supporting clinical education during the COVID 19 pandemic. Amep 11, 513-516. doi:10.2147/amep.s257750

Unity Technologies (2020). Unity. Available at: https://unity.com/ (Accessed December 10, 2020).

Wickham, H. (2016). ggplot2: Elegant Graphics for Data Analysis. New York: Springer-Verlag. 978-3-319-24277-4. https://ggplot2.tidyverse.org.

Zhaoyang, R., Sliwinski, M. J., Martire, L. M., and Smyth, J. M. (2018). Age differences in adults' daily social interactions: An ecological momentary assessment study. Psychol. Aging 33 (4), 607-618. doi:10.1037/pag0000242

Conflict of Interest: The authors declare that the research was conducted in the absence of any commercial or financial relationships that could be construed as a potential conflict of interest.

Publisher's Note: All claims expressed in this article are solely those of the authors and do not necessarily represent those of their affiliated organizations, or those of the publisher, the editors and the reviewers. Any product that may be evaluated in this article, or claim that may be made by its manufacturer, is not guaranteed or endorsed by the publisher.

Copyright (C) 2021 Wang, Xiong, Liu, Jung, Stone and Chukoskie. This is an openaccess article distributed under the terms of the Creative Commons Attribution License (CC BY). The use, distribution or reproduction in other forums is permitted, provided the original author(s) and the copyright owner(s) are credited and that the original publication in this journal is cited, in accordance with accepted academic practice. No use, distribution or reproduction is permitted which does not comply with these terms. 